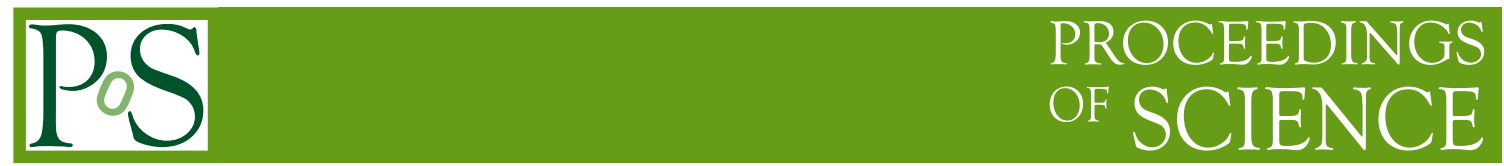

\title{
Light scalars: From lattice to the LHC via holography
}

\author{
Alex Pomarol* \\ Dept. de Física, IFAE and BIST, Universitat Autònoma de Barcelona, 08193 Bellaterra, \\ Barcelona \\ E-mail: alex.pomarol@uab.cat
}

We study the conformal to non-conformal transition in strongly-coupled theories using holography, trying to understand the presence of light scalars as recent lattice simulations seem to suggest. This can have important implications for solutions to the hierarchy problem via $\mathrm{TeV}$ strong-dynamics and their searches at the LHC.

Corfu Summer Institute 2017 'School and Workshops on Elementary Particle Physics and Gravity' 2-28 September 2017

Corfu, Greece

${ }^{*}$ Speaker. 


\section{Introduction}

We are interested here in strongly-coupled theories close to the conformal transition. This is the transition from a theory in the conformal regime to a non-conformal one. For example, in QCD, when the number of fermions is enlarged, we expect the theory to become a conformal field theory (CFT) at the IR. It is unclear where this exactly happens, but lattice simulations suggest that this could be around $N_{F} \sim 10$. There are several motivations to study strongly-coupled theories close to the conformal transition. First, recent lattice simulations suggest that, contrary to ordinary QCD, theories close to the conformal transition have as the lightest resonance a $0^{++}$scalar (apart, of course, from the goldstone bosons, the pions) [1,2]. It is unclear the origin of the lightness of this state. Some arguments suggest that this could be a dilaton, the goldstone associated to the breaking of scale invariance. For physics beyond the SM (BSM), theories close to the conformal transition are also of utmost interests. These theories allow to generate a large hierarchy of scales that could be useful to explain, for example, the difference between the electroweak scale and the Planck scale, or the differences in the SM fermion mass spectrum. Furthermore, if a light scalar is present in these theories, one could speculate whether this can be the Higgs, or whether this could be the most feasible resonance to search for at the LHC.

It has been suggested in Ref. [3] that the conformal transition is characterized by the merging of an IR fixed point with a UV fixed point. This is expected to occur when the dimensionality of a scalar operator of the theory, e.g. $q \bar{q}$ in a QCD-like theory, approaches two. In this case, conformality is lost when this operator gets a complex dimension. Holography, based on the correspondence (or duality) between strongly-coupled CFTs and weakly-coupled five-dimensional Anti-de-Sitter theories $\left(\mathrm{AdS}_{5}\right)$ [4], allows to study this transition. As we will discuss below, in the $\mathrm{AdS}_{5}$ theory the scale symmetry is lost when a scalar $\Phi$, that plays the role of the $q \bar{q}$ operator, gets a mass below the BF-bound $M_{\Phi}^{2}=-4 / L^{2}$ [3]. When this happens, the scalar becomes tachyonic and gets a non-zero profile. Working with the weakly-coupled $\mathrm{AdS}_{5}$ theory it will be possible to calculate the spectrum of resonances of the theory and see whether it contains a light scalar or not [5].

\section{A five-dimensional model for the conformal transition}

We will work within the simplest possible holographic five-dimensional model that embody the properties of strongly-coupled theories that we want to study. This is a deformed CFT with a scalar operator, $q_{L}^{i} \bar{q}_{R}^{j}\left(i, j=1, \ldots, N_{F}\right)$ for concreteness, whose dimension becomes imaginary. This means that the scalar $q_{L}^{i} \bar{q}_{R}^{j}$ gets a vacuum expectation value (VEV), signaling the lost of conformality. The global symmetry of this theory is $U\left(N_{F}\right)_{L} \otimes U\left(N_{F}\right)_{R}$ that is also broken by the VEV of the scalar $\left\langle q_{L}^{i} \bar{q}_{R}^{j}\right\rangle \propto \mathbb{1}$ down to the diagonal subgroup $U\left(N_{F}\right)_{L} \otimes U\left(N_{F}\right)_{R} \rightarrow U\left(N_{F}\right)_{V}$. This holographic model will consists in a $U\left(N_{F}\right)_{L} \otimes U\left(N_{F}\right)_{R}$ gauge theory in 5D with a complex scalar $\Phi$ transforming as a $\left(\mathbf{N}_{\mathbf{F}}, \overline{\mathbf{N}}_{\mathbf{F}}\right) .{ }^{1}$ This scalar plays the role of the $q \bar{q}$ operator whose VEV is responsible for the breaking of the conformal and gauge symmetry, and therefore its mass will be

\footnotetext{
${ }^{1}$ We could incorporate the anomaly of the $\mathrm{U}(1)_{A}$ by adding a CS-term to the $5 \mathrm{D}$ theory.
} 
related to the dimension of the $q \bar{q}$ operator through the AdS/CFT dictionary entree [4]:

$$
\operatorname{Dim}[q \bar{q}]=2+\sqrt{4+M_{\Phi}^{2}} .
$$

We also impose parity, defined as the interchange $L \leftrightarrow R$. The Lagrangian is given by

$$
\frac{1}{M_{5}} \mathscr{L}_{5}=-\frac{1}{4} \operatorname{Tr}\left[L_{M N} L^{M N}+R_{M N} R^{M N}\right]-\frac{\alpha}{4}\left(\operatorname{Tr}\left[L_{M N}+R_{M N}\right]\right)^{2}+\frac{1}{2} \operatorname{Tr}\left|D_{M} \Phi\right|^{2}-V(\Phi),
$$

with the indices running over the five dimensions, $M=\{\mu, 5\}$. We parametrize the fields as $\Phi=\Phi_{s}+T_{a} \Phi_{a}$, with $\operatorname{Tr}\left[T_{a} T_{b}\right]=\delta_{a b}$ (and similarly for $L_{M}$ and $R_{M}$ ). The fields $\Phi_{s}$ and $\Phi_{a}$ will respectively transform as a singlet and adjoint under the $U\left(N_{F}\right)_{V}$. The covariant derivative and the potential are given by

$$
D_{M} \Phi=\partial_{M} \Phi+i L_{M} \Phi-i \Phi R_{M}, \quad V(\Phi)=\frac{1}{2} M_{\Phi}^{2} \operatorname{Tr}|\Phi|^{2}+\frac{1}{4} \lambda_{1} \operatorname{Tr}|\Phi|^{4}+\frac{1}{4} \lambda_{2}\left(\operatorname{Tr}|\Phi|^{2}\right)^{2} .
$$

The 5D metric in conformal coordinates is defined as $d s^{2}=a^{2}(z)\left(\eta_{\mu v} d x^{\mu} d x^{\nu}-d z^{2}\right)$ where $a(z)$ is the warp factor. We will work within $\operatorname{AdS}_{5}: a(z)=L / z$, where $L$ is the AdS curvature radius. As explained above, our important assumption here is to consider that the conformal breaking arise from the RG-evolution of $\operatorname{Dim}[q \bar{q}]$ down to 2 where it becomes imaginary. On the AdS side, this corresponds from Eq. (2.1) to a 5D mass of $\Phi$ below the BF bound, making the AdS tachyon to turn on. For this purpose, we will take

$$
M_{\Phi}^{2}=-(4+\varepsilon) / L^{2},
$$

and work in the limit $\varepsilon \rightarrow 0$. As the mass of $\Phi$ is slightly below the BF bound, the profile of $\Phi$ turns on in the 5D bulk, breaking the conformal and chiral symmetry $U\left(N_{F}\right)_{L} \otimes U\left(N_{F}\right)_{R} \rightarrow U\left(N_{F}\right)_{V} . \Phi$ will grow as $\sim z^{2}$, as expected from a dimension-two perturbation. When the energy momentum tensor induced by the nonzero $\Phi$ profile gets of order the inverse of the 5D Newton constant, $1 / G_{N}$, the backreaction on the metric will be important, starting to depart then from AdS, and signaling the breaking of the conformal symmetry. Instead of considering the change in the metric, that will complicate and then obscure our results, we will take the simplified assumption that the growth of the $5 \mathrm{D}$ tachyon is regularized by an IR-brane at some point in the AdS throat $z=z_{\mathrm{IR}}$. This will be determined dynamically by minimizing with respect $1 / z_{\mathrm{IR}}$, as this corresponds to the VEV of a dynamical field, the radion, or the corresponding dilaton in the dual theory (see section 2.3). We consider that the IR-boundary will capture in a simple way the effect of the metric feedback that will be generically parametrized by the boundary terms. In particular, this means that $\Phi$ might also have a potential on the IR-boundary. For this reason, we will consider the presence of a mass term on the IR-boundary: $\mathscr{L}_{\mathrm{IR}}=a^{4} m_{b}^{2} \operatorname{Tr}|\Phi|^{2} /\left.2\right|_{z_{\mathrm{IR}}}$, and study its impact on the properties of the model. As it is usual in AdS/CFT, we will be regularizing the UV-divergencies by placing a UV-boundary at $z=z_{\mathrm{UV}}$ and taking the limit $z_{\mathrm{UV}} \rightarrow 0$ at the end of the calculation of physical quantities.

By the AdS/CFT correspondence, the mass-parameter $M_{5}$, that corresponds to the inverse of the 5D gauge coupling squared, is related to the large- $N_{c}$ expansion parameter of the dual stronglycoupled CFT: $1 /\left(M_{5} L\right) \sim 16 \pi^{2} / N_{c}$. In this correspondence 5D double-trace operators are suppressed with respect to single-trace ones, i.e.,

$$
\alpha \sim \lambda_{2} / \lambda_{1} \sim 1 / N_{c}
$$


For this reason these terms were neglected in previous holographic approaches to QCD [6, 7]. Nevertheless, the parameters $\alpha$ and $\lambda_{2}$ are accompanied by a factor $N_{F}$ and then their effects are not suppressed for large values of $N_{F}$. Therefore it is important to keep double-trace operators in Eq. (2.2) when comparing our results to strongly-coupled theories in the large $N_{c}$ and $N_{F}$ limit. In particular, $\lambda_{2}$ will be responsible to generate a mass splitting in the scalar sector between the singlet $\left(\Phi_{s}\right)$ and the adjoint states $\left(\Phi_{a}\right)$, as it is observed in lattice results with large $N_{F}[1,2]$.

It is important to remark that we cannot consider the strict limit $N_{F} \sim N_{c}$ in our 5D model. In this limit loops of vector or scalar resonances contribute as $\frac{N_{F}}{M_{5} L} \frac{1}{16 \pi^{2}} \sim N_{F} / N_{c} \sim 1$, meaning that we cannot perform a perturbative expansion since the $5 \mathrm{D}$ theory is strongly-coupled. Therefore the 5D theory will be only reliable if we approach the large- $N_{F}$ and large- $N_{c}$ limit $\left(M_{5} L \rightarrow \infty\right)$ keeping $N_{F} \ll 16 \pi^{2} M_{5} L$. Basically, the only difference here with respect to previous models for holographic QCD is the non-negligible presence of $\alpha$ and $\lambda_{2}$.

\subsection{The tachyon solution}

The non-zero profile for $\Phi$ will be taken to be along the $\phi=\left|\Phi_{s}\right|$ direction. Since we will be interested in the solution close to the conformal transition, we will take the limit $\varepsilon \rightarrow 0$. Therefore the solution for $\phi$ only depends on $z_{\mathrm{IR}}, \lambda$ and $m_{b}$. At the UV-boundary we will impose $\phi=0$, otherwise we would be breaking the chiral symmetry from UV-physics (as adding an explicit mass term to the quarks in the dual theory). On the other hand, at the IR-boundary, we must impose the boundary condition determined by the model above: $\left.z_{\mathrm{IR}} \partial_{5} \phi\right|_{z_{\mathrm{IR}}}=-\left.m_{b}^{2} \phi\right|_{z_{\mathrm{IR}}}$. This boundary condition however cannot be satisfied for all values of $z_{\mathrm{IR}}$ (unless $\phi=0$ ), meaning that the tachyon can only turn on if the IR-boundary is beyond some critical value, $z_{\mathrm{IR}}>z_{\mathrm{IR}}^{c}$. It is easy to find this value $z_{\mathrm{IR}}^{c}$, just by looking for the place where the IR-boundary must be placed in order to have a 4D massless mode. For this to happen, the wave-function of this massless mode must satisfy the linearized bulk EOM with $p^{2}=0$. We obtain

$$
\phi(z)=A z^{2} \sin \left(\sqrt{\varepsilon} \ln \frac{z}{z_{\mathrm{UV}}}\right),
$$

where $A$ is a normalization constant, and where the IR-boundary condition at $z_{\mathrm{IR}}=z_{\mathrm{IR}}^{c}$ leads to

$$
\sqrt{\varepsilon} \ln \frac{z_{\mathrm{IR}}^{c}}{z_{\mathrm{UV}}}=n \pi, n=1,2, \ldots,
$$

after taking the formal limit $\varepsilon \rightarrow 0$ (and $z \mathrm{UV} \rightarrow 0$ ). The presence of $n$ solutions is a well-known feature of this configurations, and it is associated to the existence of Efimov states. We will be considering $n=1$, that will give us the global minimum, being the other possibilities just local minima.

For $z_{\mathrm{IR}}>z_{\mathrm{IR}}^{c}$, the above massless mode will have a negative mass, becoming a tachyon, and then getting a nonzero profile and triggering the breaking of conformal and chiral symmetry breaking. We can distinguish two limits:

1. For $z_{\mathrm{IR}} \gtrsim z_{\mathrm{IR}}^{c}$, the tachyon profile is very close to Eq. (2.6). 

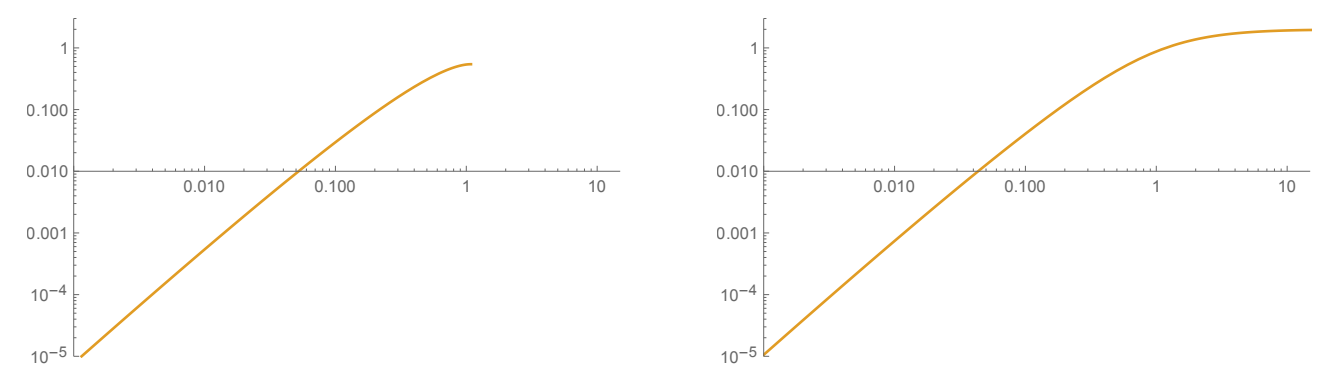

Figure 1: $5 D$ tachyon solutions for case (1) with $z_{\mathrm{IR}}=1.2 z_{\mathrm{IR}}^{c}($ left $)$, and case (2) with $z_{\mathrm{IR}}=20 z_{\mathrm{IR}}^{c}$ (right). We have taken $\lambda=1$ and $m_{b}=0$.

2. For $z_{\mathrm{IR}} \gg z_{\mathrm{IR}}^{c}$, the tachyon profile grows till reaching a maximum value determined by the minimum of the $5 \mathrm{D}$ potential $V(\Phi)$, i.e., $\phi_{\max }=\sqrt{M_{\Phi}^{2} / \lambda}$.

Both types of configurations are shown in Fig. 1. In the figure in the left we have taken $z_{\mathrm{IR}} \approx z_{\mathrm{IR}}^{c}$, while the one in the right $z_{\mathrm{IR}}^{c} \gg z_{\mathrm{IR}}$. The model can provide both configurations, as $z_{\mathrm{IR}}$ can be dynamically settled, depending on $m_{b}$, either relatively close to $z_{\mathrm{IR}}^{c}$ or to much larger values. The scale $1 / z_{\mathrm{IR}}^{c}$ corresponds to the scale of chiral symmetry breaking, while $1 / z_{\mathrm{IR}}$ is the scale of confinemet. Therefore, while in case (1) both scales are similar, in case (2) the scale of chiral breaking is much larger than the scale of confinement. Indeed, in case (2) the theory below $1 / z_{\mathrm{IR}}^{c}$ enters into another CFT where the (gauge) symmetry is just $S U\left(N_{F}\right)_{V}$ with $\Phi_{s}$ and $\Phi_{a}$ respectively in the singlet and Adjoint representation. Their squared masses are given by $\sim 8 / L^{2}$ and $8\left(1-2 \lambda_{2} /(3 \lambda)\right) / L^{2}$ respectively. This corresponds in the dual theory to $q \bar{q}$ operators of very high dimension (becoming irrelevant). In this new CFT, scale invariance is broken at $1 / z_{\mathrm{IR}}$.

\subsection{Excitations around the 5D tachyon}

We will start studying the spectrum for a fixed value of the IR-boundary, $z_{\mathrm{IR}}$, to discuss later the properties of the dilaton, whose mass will be determined by minimizing the energy with respect to $z_{\mathrm{IR}}$. The purpose is to show the properties of the spectrum as we increase $z_{\mathrm{IR}}$ and move from scenario (1) to (2). The results are presented in the left plot of Fig. 2 as a function of $z_{\mathrm{IR}} / z_{\mathrm{IR}}^{c}$ and for $\lambda=1, \lambda_{2}=-3 \lambda$ and $m_{b}=0$ (solid line) and $m_{b}=-1$ (dashed line). Following the notation used in QCD, we refer by $f_{0}, a_{0}, \rho$ and $a_{1}$ respectively the singlet-scalar, adjoint-scalar, vector and axial-vector resonances. Since $F_{\pi}$ is the only quantity that depends on $M_{5}\left(N_{c}\right.$ in the dual theory), we have fixed its value following [7]. We have normalized the spectrum to $m_{\rho}$. From the left figure of Fig. 2 we see that for $z_{\mathrm{IR}} \sim z_{\mathrm{IR}}^{c}$ we are in the scenario (1) where the chiral breaking scale is smaller or of order the confinement scale. This is reflected in the $\rho-a_{1}$ mass splitting that is always small. As we increase $z_{\mathrm{IR}} / z_{\mathrm{IR}}^{c}$, we move towards scenario (2) where the breaking of the chiral symmetry is larger, as can be appreciated by the growth of $F_{\pi}$ and the $\rho-a_{1}$ splitting. For $z_{\mathrm{IR}} \gg z_{\mathrm{IR}}^{c}$ the theory is close to a different CFT, the one discussed before, where the global group is $U\left(N_{F}\right)$ and the scalars and axial-vector have masses larger than $1 / z_{\mathrm{IR}}$.

Another important prediction of the proposed 5D model is that only the scalar sector presents 

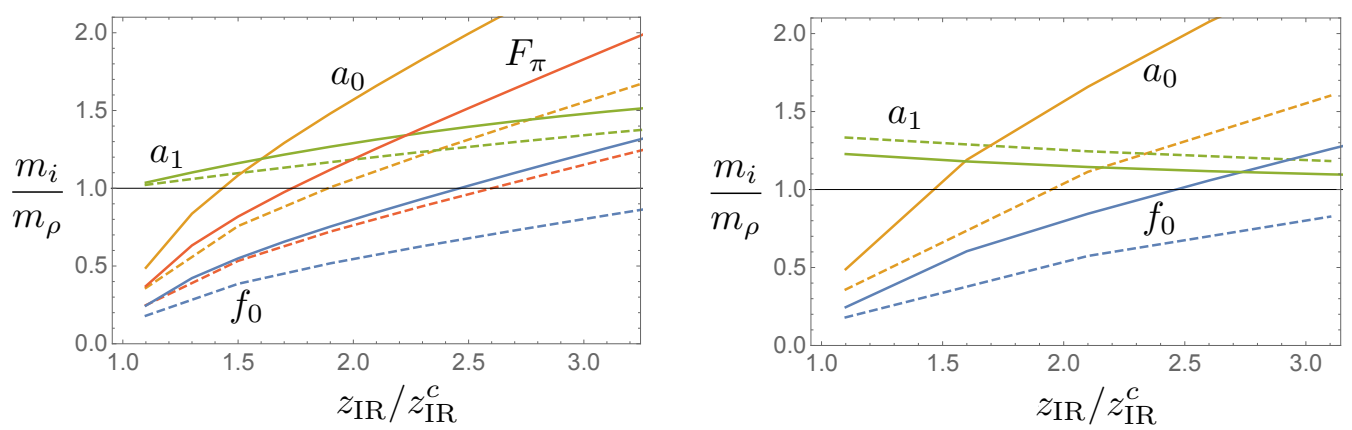

Figure 2: Resonance mass spectrum as a function of the position of the IR-boundary, either for constant $\lambda$ (left) or constant $F_{\pi}$ (right), for two values of the boundary mass: $m_{b}=0$ (solid line) and $m_{b}=-1$ (dashed line).

a mass splitting between the singlet $\left(f_{0}\right)$ and the adjoint $\left(a_{0}\right)$. This splitting is generated by $\lambda_{2}$. In the gauge sector the only physical difference between the singlet and adjoint arises from $\alpha$ in Eq. (2.2). This however does not produce any mass splitting in the vector sector $V_{M}$ between the the singlet resonances (the $\omega$ in QCD) and the adjoint resonances $(\rho)$, as these do not depend on $\alpha$ but only on the boundary conditions on the IR-boundary that are the same for all gauge fields. For the axial-vector $A_{M}$ a mass splitting could arise from their coupling to $\Phi$ that, for $\alpha \neq 0$, is different for the singlet and adjoint. Nevertheless, when fitting the model to the UV, as we do here, one obtains $\alpha=0$ [7]. Therefore the proposed 5D model predict that the only mass splitting between the singlet and adjoint resonances (the Kaluza-Klein states) can only be possible in the scalar sector. Of course, these mass splittings could be generated at the loop level or from higherdimensional operators in Eq. (2.2), but this are suppressed by the 5D cutoff (scale at which the 5D theory becomes strongly coupled) $\Lambda_{5} \lesssim 24 \pi^{3} M_{5}$.

It is more instructive, also to compare later our results with lattice simulations, to analyze the spectrum at equal $F_{\pi}$. For this purpose, we adjust $\lambda$ to fulfill, for the different values of $z_{\mathrm{IR}} / z_{\mathrm{IR}}^{c}$, the relation $F_{\pi} \sim m_{\rho} / 8$ as in QCD. This can always be achieved as $F_{\pi}$ roughly scale as $1 / \sqrt{\lambda}$. The results are given in the right plot of Fig. 2. For $z_{\mathrm{IR}} \gg z_{\mathrm{IR}}^{c}$, however, we must increase $\lambda$ to a too large values, putting in danger the perturbative approach. We find the for $m_{b}=0\left(m_{b}=-1\right)$, in order to stay with $\lambda \leq 4$, we must have $z_{\mathrm{IR}} / z_{\mathrm{IR}}^{c} \lesssim 2.6\left(z_{\mathrm{IR}} / z_{\mathrm{IR}}^{c} \lesssim 4.1\right)$. Keeping in this region, we find that the scalar $f_{0}$ is the lightest resonance. A reason for the relative lightness of the scalar excitation is the following. As we approach the conformal edge, the corresponding dimension of the scalar operator becomes close to 2 . This is the lowest value before being imaginary. As it is well-known, the dimension of a scalar operator has a minimal value determined by its unitarity bound, in this case $\operatorname{Dim}[q \bar{q}]=1$, a limit in which the scalar decouple from the CFT. Therefore it is expected that, as the scalar approach this limit, the mass of the lightest resonance becomes smaller.

\subsection{Dilaton mass}

Since $1 / z_{\mathrm{IR}}$ is a dynamical field, the radion, that in the dual 4D CFT corresponds to the dilaton, its value must be determined by its EOM. Interestingly, the 5D tachyon provide a minimum for 
$z_{\mathrm{IR}}$ due to its logarithmic dependence (see Eq. (2.6)). The minimization condition can be read from the junction condition following Ref. [8]. From there we can get the mass of the dilaton: $m_{D}^{2} \propto L\left(m_{b}^{2} L^{2}+2\right)^{3} / \sqrt{\lambda} \times \partial_{z_{\mathrm{IR}}} \phi\left(z_{\mathrm{IR}}\right) / z_{\mathrm{IR}}$. From this equation, it is clear that only when the value of the tachyon on the IR-boundary mildly depends on the IR-boundary position, we can expect a light dilaton. From Fig. 1, this only happens for large $z_{\mathrm{IR}} / z_{\mathrm{IR}}^{c}$, but in this case the chiral breaking is large. Lattice simulations do not seem to see a large breaking of the chiral symmetry when approaching the conformal transition, therefore we can conclude that the light $0^{++}$state is not expected to be the dilaton.

\section{Lattice QCD in the large $N_{F}$}

Lattice results for QCD with $N_{F}=8$ have been reported in Ref. [1, 2]. At such large value of $N_{F}$, it is believed that QCD is close to the conformal transition, expected to occur around $N_{F} \sim 10$. It was found [1,2]

$$
F_{\pi} \simeq 0.14 m_{\rho}, m_{f_{0}} \simeq 0.5 m_{\rho}, m_{a_{0}} \simeq m_{\rho}, m_{a_{1}} \simeq 1.4 m_{\rho} .
$$

As compare to real QCD these values show a lighter $f_{0}$ scalar and a smaller mass splitting between the vector and axial-vector resonance. Surprisingly, the ratio of $F_{\pi} / m_{\rho}$ is quite similar to real QCD, showing that this quantity is quite independent of $N_{F}$. Let us compare our results to the values of Eq. (3.1). From the right plot of Fig. 2, where we fixed $F_{\pi}=m_{\rho} / 8$ and used the matching to the two-point vector-vector correlator in the UV following [7], we see that for values of $z_{\mathrm{IR}} / z_{\mathrm{IR}}^{c} \lesssim 2$ our predictions on the spectrum of resonances follows quite close to the pattern given in Eq. (3.1). This suggests that the light scalar found in lattice simulations could mostly be a meson scalar $q \bar{q}$, with its lightness arising from the fact that $\operatorname{Dim}[q \bar{q}] \sim 2$. Another prediction from the holographic model is the small mass splitting between adjoint and singlet in the vector and axial-vector sector. It would be interesting to check this prediction from lattice simulations.

\section{Implications for BSM}

The model described here open new possibilities for physics beyond the SM. First, it can solve the big hierarchy problem, since by choosing small values of $\varepsilon$, we can generate a small IR scale from a large UV scale. Indeed, from Eq. (2.7) we have

$$
\frac{1}{z_{\mathrm{IR}}} \sim \frac{1}{z_{\mathrm{IR}}^{c}}=e^{-\pi / \sqrt{\varepsilon}} \frac{1}{z_{\mathrm{UV}}} \ll \frac{1}{z_{\mathrm{UV}}} .
$$

Furthermore, the presence of a light scalar $f_{0}$ in the spectrum raises the old question of whether this resonance could be identified with the Higgs $h$. In the holographic model we can calculate the couplings of $f_{0}$ to the SM fields. The result is shown in Fig. 3. As expected, the couplings tend to the SM values as we decrease the dimension of $q \bar{q}$ and approach the decoupling limit where $f_{0}$ becomes closer to an elementary field. Nevertheless, for $\operatorname{Dim}[q \bar{q}] \sim 2$ the departures from the SM are still too large to properly fit the present experimental values. 

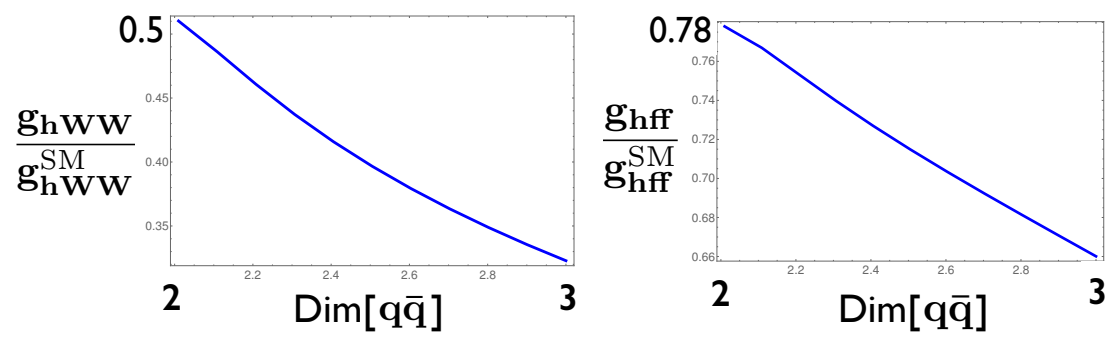

Figure 3: Couplings of the $f_{0}$ resonance to $S M$ gauge bosons and fermions as a function of the dimension of the associated operator in the dual theory using Eq. (2.1).

For models in which the Higgs arises as a composite pseudo-goldstone boson, that have been extensively searched for at the LHC, the presence of a light scalar $f_{0}$ has also important implications. Being $f_{0}$ the lightest resonance, it implies that other resonances will mostly decay to $f_{0}$, that sequentially will decay to tops or $W_{L}, Z_{L}, h$. As a consequence LHC search strategies must be changed. For example, searches for top partners $T$ must look for three SM particles in the final states instead of two, as we could have, for example, $T \rightarrow t f_{0} \rightarrow t \bar{t} t$. Finally, the fact that the Higgs operator $q \bar{q}$ has dimension close to 2 allows to alleviate the flavor limits on this type of models, similarly as in walking technicolor. In particular, flavor models as the one proposed in Ref. [9] can satisfy all flavor and CP-violating constraints for an IR scale of few TeV.

\section{Conclusions}

Using holography we have studied the implications of strongly-coupled theories close to the conformal transition following the approach of Ref. [3]. We have seen that the theory predicts a scalar $0^{++}$as the lightest resonance. Nevertheless, this scalar is not the dilaton but a scalar whose interpolating operator $q \bar{q}$ gets the lowest possible dimension. Furthermore, the mass of this scalar cannot be parametrically much smaller than the mass gap of the theory. This could be checked by lattice simulations. Being the scalar the lightest resonance have important implications for the LHC, as other resonances (vectorial of fermionic) will decay to it with BR of order one. Therefore LHC search strategies must be optimized differently from present ones.

\section{Acknowledgements}

I would like to thank my collaborators L. Salas and O. Pujolas that contributed to the work reported here. This work was supported by the Catalan ICREA Academia Program and FPA201455613-P, 2014-SGR-1450 and SO-2012-0234. 


\section{References}

[1] Y. Aoki et al. [LatKMI Collaboration], Phys. Rev. D 96 (2017) no.1, 014508; Phys. Rev. D 89 (2014) 111502 .

[2] R. C. Brower, A. Hasenfratz, C. Rebbi, E. Weinberg and O. Witzel, Phys. Rev. D 93 (2016) no.7, 075028.

[3] D. B. Kaplan, J. W. Lee, D. T. Son and M. A. Stephanov, Phys. Rev. D 80 (2009) 125005.

[4] J. M. Maldacena, Adv. Theor. Math. Phys. 2 (1998) 231; S. S. Gubser, I. R. Klebanov and A. M. Polyakov, Phys. Lett. B 428 (1998) 105; E. Witten, Adv. Theor. Math. Phys. 2 (1998) 253.

[5] L. Salas, A. Pomarol and O. Pujolas in preparation.

[6] J. Erlich, E. Katz, D. T. Son and M. A. Stephanov, Phys. Rev. Lett. 95 (2005) 261602.

[7] L. Da Rold and A. Pomarol, Nucl. Phys. B 721 (2005) 79; JHEP 0601 (2006) 157.

[8] C. Csaki, M. L. Graesser and G. D. Kribs, Phys. Rev. D 63 (2001) 065002.

[9] G. Panico and A. Pomarol, JHEP 1607 (2016) 097. 